\title{
A CHARACTERISTIC ZERO HILBERT-KUNZ CRITERION FOR SOLID CLOSURE IN DIMENSION TWO
}

\author{
Holger Brenner
}

\begin{abstract}
Let $I$ denote a homogeneous $R_{+}$-primary ideal in a two-dimensional normal standard-graded domain over an algebraically closed field of characteristic zero. We show that a homogeneous element $f$ belongs to the solid closure $I^{\star}$ if and only if $e_{H K}(I)=e_{H K}((I, f))$, where $e_{H K}$ denotes the Hilbert-Kunz multiplicity of an ideal, introduced here in characteristic zero in the graded dimension two case. This provides a version in characteristic zero of the well-known HilbertKunz criterion for tight closure in positive characteristic.
\end{abstract}

\section{Introduction}

Let $(R, \mathfrak{m})$ denote a local Noetherian ring or an $\mathbb{N}$-graded algebra of dimension $d$ of positive characteristic $p$. Let $I$ denote an $\mathfrak{m}$-primary ideal, and set $I^{[q]}=$ $\left(f^{q}: f \in I\right)$ for a prime power $q=p^{e}$. Then the Hilbert-Kunz function of $I$ is given by

$$
e \longmapsto \lambda\left(R / I^{\left[p^{e}\right]}\right)
$$

where $\lambda$ denotes the length. The Hilbert-Kunz multiplicity of $I$ is defined as the limit

$$
e_{H K}(I)=\lim _{e \rightarrow \infty} \lambda\left(R / I^{\left[p^{e}\right]}\right) / p^{e d} .
$$

This limit exists as a positive real number, as shown by Monsky in [9]. It is an open question whether this number is always rational.

The Hilbert-Kunz multiplicity is related to the theory of tight closure. Recall that the tight closure of an ideal $I$ in a Noetherian ring of characteristic $p$ is by definition the ideal

$$
I^{*}=\left\{f \in R: \exists c \text { not in any minimal prime }: c f^{q} \in I^{[q]} \text { for almost all } q=p^{e}\right\} .
$$

For an analytically unramified and formally equidimensional local ring $R$ the equation $e_{H K}(I)=e_{H K}(J)$ holds if and only if $I^{*}=J^{*}$ holds true for ideals $I \subseteq J$ (see [6, Theorem 5.4]). Hence $f \in I^{*}$ if and only if $e_{H K}(I)=e_{H K}((I, f))$. This is the Hilbert-Kunz criterion for tight closure in positive characteristic.

The aim of this paper is to give a characteristic zero version of this relationship between Hilbert-Kunz multiplicity and tight closure for $R_{+}$-primary homogeneous ideals in a normal two-dimensional graded domain $R$. There are several

Received March 18, 2004.

2000 Mathematics Subject Classification. 13A35; 13D40; $14 \mathrm{H} 60$. 
notions for tight closure in characteristic zero, defined either by reduction to positive characteristic or directly. We will work with the notion of solid closure (see [5]). In dimension two, the containment in the solid closure $f \in\left(f_{1}, \ldots, f_{n}\right)^{\star}$ means that the open subset $D(\mathfrak{m}) \subset \operatorname{Spec} A$ is not an affine scheme, where $A=R\left[T_{1}, \ldots, T_{n}\right] /\left(f_{1} T_{1}+\ldots+f_{n} T_{n}+f\right)$ is the so-called forcing algebra, see [1, Proposition 1.3].

The definition of the Hilbert-Kunz multiplicity in positive characteristic does not suggest at first sight an analogous notion in characteristic zero. However, a bridge is provided by the following result of [2], which gives an explicit formula for the Hilbert-Kunz multiplicity and proves its rationality in dimension two (the rationality of the Hilbert-Kunz multiplicity for the maximal ideal was also obtained independently in [10]).

Theorem 1. Let $R$ denote a two-dimensional standard-graded normal domain over an algebraically closed field of positive characteristic, $Y=\operatorname{Proj} R$. Let $I=$ $\left(f_{1}, \ldots, f_{n}\right)$ denote a homogeneous $R_{+}$-primary ideal generated by homogeneous elements $f_{i}$ of degree $d_{i}, i=1, \ldots, n$. Then the Hilbert-Kunz multiplicity of the ideal $I$ equals

$$
e_{H K}(I)=\frac{\operatorname{deg}(Y)}{2}\left(\sum_{k=1}^{t} r_{k} \nu_{k}^{2}-\sum_{i=1}^{n} d_{i}^{2}\right)
$$

Here the numbers $r_{k}$ and $\nu_{k}$ come from the strong Harder-Narasimhan filtration of the syzygy bundle $\operatorname{Syz}\left(f_{1}^{q}, \ldots, f_{n}^{q}\right)(0)$ given by the short exact sequence

$$
0 \longrightarrow \operatorname{Syz}\left(f_{1}^{q}, \ldots, f_{n}^{q}\right)(0) \longrightarrow \bigoplus_{i=1}^{n} \mathcal{O}\left(-q d_{i}\right) \stackrel{f_{1}^{q}, \ldots, f_{n}^{q}}{\longrightarrow} \mathcal{O}_{Y} \longrightarrow 0
$$

This syzygy bundle is a locally free sheaf on the smooth projective curve $Y=$ $\operatorname{Proj} R$, and its strong Harder-Narasimhan filtration is a filtration $\mathcal{S}_{1} \subset \ldots \subset$ $\mathcal{S}_{t}=\operatorname{Syz}\left(f_{1}^{q}, \ldots, f_{n}^{q}\right)(0)$ such that the quotients $\mathcal{S}_{k} / \mathcal{S}_{k-1}$ are strongly semistable, meaning that every Frobenius pull-back is semistable. Such a filtration exists for $q$ big enough by a theorem of Langer, [8, Theorem 2.7]. Then we set $r_{k}=$ $\operatorname{rk}\left(\mathcal{S}_{k} / \mathcal{S}_{k-1}\right)$ and $\nu_{k}=-\mu\left(\mathcal{S}_{k} / \mathcal{S}_{k-1}\right) / q \operatorname{deg}(Y)$, where $\mu$ denotes the slope.

To define the Hilbert-Kunz multiplicity in characteristic zero we now take the right hand side of the above formula as our model.

Definition 1. Let $R$ denote a two-dimensional normal standard-graded $K$-domain over an algebraically closed field $K$ of characteristic zero. Let $I=$ $\left(f_{1}, \ldots, f_{n}\right)$ be a homogeneous $R_{+}$-primary ideal given by homogeneous ideal generators $f_{i}$ of degree $d_{i}$. Let $\mathcal{S}_{1} \subset \ldots \subset \mathcal{S}_{t}=\operatorname{Syz}\left(f_{1}, \ldots, f_{n}\right)(0)$ denote the Harder-Narasimhan filtration of the syzygy bundle on $Y=\operatorname{Proj} R$, set $\mu_{k}=$ $\mu\left(\mathcal{S}_{k} / \mathcal{S}_{k-1}\right)$ and $r_{k}=\operatorname{rk}\left(\mathcal{S}_{k} / \mathcal{S}_{k-1}\right)$. Then the Hilbert-Kunz multiplicity of $I$ is by definition

$e_{H K}(I)=\frac{\operatorname{deg}(Y)}{2}\left(\sum_{k=1}^{t} r_{k}\left(\frac{\mu_{k}}{\operatorname{deg}(Y)}\right)^{2}-\sum_{i=1}^{n} d_{i}^{2}\right)=\frac{\sum_{k=1}^{t} r_{k} \mu_{k}^{2}-\operatorname{deg}(Y)^{2} \sum_{i=1}^{n} d_{i}^{2}}{2 \operatorname{deg}(Y)}$ 
It is easy to show that this definition does not depend on the chosen ideal generators and is therefore an invariant of the ideal, see [2, Proposition 4.9]. With this invariant we can in fact give the following Hilbert-Kunz criterion for solid closure in characteristic zero in dimension two (see Theorem 3.3):

Theorem 2. Let $K$ denote an algebraically closed field of characteristic zero, let $R$ denote a standard-graded two-dimensional normal $K$-domain. Let $I$ be a homogeneous $R_{+}$-primary ideal and let $f$ denote a homogeneous element. Then $f$ is contained in the solid closure, $f \in I^{\star}$, if and only if $e_{H K}(I)=e_{H K}((I, f))$.

To prove this theorem it is convenient to consider more generally for a locally free sheaf $\mathcal{S}$ on a smooth projective curve $Y$ the expression

$$
\mu_{H K}(\mathcal{S})=\sum_{k=1}^{t} r_{k} \mu_{k}^{2},
$$

where $r_{k}$ and $\mu_{k}$ are the ranks and the slopes of the semistable quotient sheaves in the Harder-Narasimhan filtration of $\mathcal{S}$. We call this number the HilbertKunz slope of $\mathcal{S}$. With this notion the Hilbert-Kunz multiplicity of an ideal $I=\left(f_{1}, \ldots, f_{n}\right)$ is related to the Hilbert-Kunz slope of the syzygy bundle by

$$
e_{H K}\left(\left(f_{1}, \ldots, f_{n}\right)\right)=\frac{1}{2 \operatorname{deg}(Y)}\left(\mu_{H K}\left(\operatorname{Syz}\left(f_{1}, \ldots, f_{n}\right)(0)\right)-\mu_{H K}\left(\bigoplus_{i=1}^{n} \mathcal{O}\left(-d_{i}\right)\right)\right) \text {. }
$$

With this notion we will in fact prove the following theorem, which implies Theorem 2 (see Theorem 2.6).

Theorem 3. Let $Y$ denote a smooth projective curve over an algebraically closed field of characteristic 0 . Let $\mathcal{S}$ denote a locally free sheaf on $Y$ and let $c \in H^{1}(Y, \mathcal{S})$ denote a cohomology class giving rise to the extension $0 \rightarrow \mathcal{S} \rightarrow$ $\mathcal{S}^{\prime} \rightarrow \mathcal{O}_{Y} \rightarrow 0$ and the affine-linear torsor $\mathbb{P}\left(\mathcal{S}^{\prime \vee}\right)-\mathbb{P}\left(\mathcal{S}^{\vee}\right)$. Then $\mathbb{P}\left(\mathcal{S}^{\prime \vee}\right)-\mathbb{P}\left(\mathcal{S}^{\vee}\right)$ is an affine scheme if and only if $\mu_{H K}\left(\mathcal{S}^{\prime}\right)<\mu_{H K}(\mathcal{S})$.

\section{The Hilbert-Kunz slope of a vector bundle}

We recall briefly some notions for locally free sheaves (or vector bundles), see [7] or [4]. Let $Y$ denote a smooth projective curve over an algebraically closed field and let $\mathcal{S}$ denote a locally free sheaf of rank $r$. Then $\operatorname{deg}(\mathcal{S})=$ $\operatorname{deg}\left(\bigwedge^{r} \mathcal{S}\right)$ is called the degree of $\mathcal{S}$ and $\mu(\mathcal{S})=\operatorname{deg}(\mathcal{S}) / r$ is called the slope of $\mathcal{S}$. If $\mu(\mathcal{T}) \leq \mu(\mathcal{S})$ holds for every locally free subsheaf $\mathcal{T} \subseteq \mathcal{S}$, then $\mathcal{S}$ is called semistable. In general there exists the so-called Harder-Narasimhan filtration. This is a filtration of locally free subsheaves $\mathcal{S}_{1} \subset \ldots \subset \mathcal{S}_{t}=\mathcal{S}$ such that the quotient sheaves $\mathcal{S}_{k} / \mathcal{S}_{k-1}$ are semistable locally free sheaves with decreasing slopes $\mu_{1}>\ldots>\mu_{t}$. The Harder-Narasimhan filtration is uniquely determined by these properties. $\mathcal{S}_{1}$ is called the maximal destabilizing subsheaf, $\mu_{1}=\mu_{\max }(\mathcal{S})$ is called the maximal slope of $\mathcal{S}$ and $\mu_{t}=\mu_{\min }(\mathcal{S})$ is called the minimal slope of $\mathcal{S}$. If $\mathcal{S} \rightarrow \mathcal{T}$ is a non-trivial sheaf homomorphism, then $\mu_{\min }(\mathcal{S}) \leq \mu_{\max }(\mathcal{T})$. 
We begin with the definition of the Hilbert-Kunz slope of $\mathcal{S}$.

Definition 1.1. Let $\mathcal{S}$ denote a locally free sheaf on a smooth projective curve over an algebraically closed field of characteristic 0 . Let $\mathcal{S}_{1} \subset \ldots \subset \mathcal{S}_{t}=\mathcal{S}$ denote the Harder-Narasimhan filtration of $\mathcal{S}$, set $r_{k}=\operatorname{rk}\left(\mathcal{S}_{k} / \mathcal{S}_{k-1}\right)$ and $\mu_{k}=$ $\mu\left(\mathcal{S}_{k} / \mathcal{S}_{k-1}\right)$. We define the Hilbert-Kunz slope of $\mathcal{S}$ by

$$
\mu_{H K}(\mathcal{S})=\sum_{k=1}^{t} r_{k} \mu_{k}^{2}=\sum_{k=1}^{t} \frac{\operatorname{deg}\left(\mathcal{S}_{k} / \mathcal{S}_{k-1}\right)^{2}}{r_{k}} .
$$

The only justification for considering this number is Theorem 3.3 below. We gather together some properties of this notion in the following proposition.

Proposition 1.2. Let $\mathcal{S}$ denote a locally free sheaf on a smooth projective curve over an algebraically closed field of characteristic 0 . Then the following hold true.

(i) If $\mathcal{S}$ is semistable, then $\mu_{H K}(\mathcal{S})=\operatorname{deg}(\mathcal{S})^{2} / \operatorname{rk}(\mathcal{S})$.

(ii) Let $\mathcal{T} \subset \mathcal{S}$ denote a locally free subsheaf occurring in the Harder-Narasimhan filtration of $\mathcal{S}$. Then $\mu_{H K}(\mathcal{S})=\mu_{H K}(\mathcal{T})+\mu_{H K}(\mathcal{S} / \mathcal{T})$.

(iii) We have $\mu_{H K}(\mathcal{S} \oplus \mathcal{T})=\mu_{H K}(\mathcal{S})+\mu_{H K}(\mathcal{T})$.

(iv) $\mu_{H K}(\mathcal{S})=\mu_{H K}\left(\mathcal{S}^{\vee}\right)$.

(v) Let $\mathcal{L}$ denote an invertible sheaf. Then

$$
\mu_{H K}(\mathcal{S} \otimes \mathcal{L})=\mu_{H K}(\mathcal{S})+2 \operatorname{deg}(\mathcal{S}) \operatorname{deg}(\mathcal{L})+\operatorname{rk}(\mathcal{S}) \operatorname{deg}(\mathcal{L})^{2} .
$$

(vi) Let $\varphi: Y^{\prime} \rightarrow Y$ denote a finite morphism between smooth projective curves of degree $n$. Then $\mu_{H K}\left(\varphi^{*}(\mathcal{S})\right)=n^{2} \mu_{H K}(\mathcal{S})$.

Proof. (i) and (ii) are clear from the definition. (iii). The maximal destabilizing subsheaf of $\mathcal{S} \oplus \mathcal{T}$ is either $\mathcal{S}_{1} \oplus 0,0 \oplus \mathcal{T}_{1}$ or $\mathcal{S}_{1} \oplus \mathcal{T}_{1}$. Hence the result follows from (ii) by induction on the rank of $\mathcal{S} \oplus \mathcal{T}$.

(iv). Let $0=\mathcal{S}_{0} \subset \mathcal{S}_{1} \subset \ldots \subset \mathcal{S}_{t}=\mathcal{S}$ denote the Harder-Narasimhan filtration of $\mathcal{S}$. Set $\mathcal{Q}_{k}=\mathcal{S} / \mathcal{S}_{k}$. This gives a filtration $0 \subset \mathcal{Q}_{t-1}^{\vee} \subset \ldots \subset$ $\mathcal{Q}_{1}^{\vee} \subset \mathcal{Q}_{0}^{\vee}=\mathcal{S}^{\vee}$. From $0 \rightarrow \mathcal{S}_{k} / \mathcal{S}_{k-1} \rightarrow \mathcal{S} / \mathcal{S}_{k-1} \rightarrow \mathcal{S} / \mathcal{S}_{k} \rightarrow 0$ we get $0 \rightarrow$ $\mathcal{Q}_{k}^{\vee} \rightarrow \mathcal{Q}_{k-1}^{\vee} \rightarrow \mathcal{Q}_{k-1}^{\vee} / \mathcal{Q}_{k}^{\vee} \cong\left(\mathcal{S}_{k} / \mathcal{S}_{k-1}\right)^{\vee} \rightarrow 0$. Hence the filtration is the Harder-Narasimhan filtration of $\mathcal{S}^{\vee}$ and the result follows from $\mu\left(\mathcal{Q}_{k-1}^{\vee} / \mathcal{Q}_{k}^{\vee}\right)=$ $-\mu\left(\mathcal{S}_{k} / \mathcal{S}_{k-1}\right)$.

(v). The Harder-Narasimhan filtration of $\mathcal{S} \otimes \mathcal{L}$ is $\mathcal{S}_{1} \otimes \mathcal{L} \subset \ldots \subset \mathcal{S}_{t} \otimes \mathcal{L}$ and $\mu\left(\mathcal{S}_{k} \otimes \mathcal{L} / \mathcal{S}_{k-1} \otimes \mathcal{L}\right)=\mu\left(\left(\mathcal{S}_{k} / \mathcal{S}_{k-1}\right) \otimes \mathcal{L}\right)=\mu\left(\mathcal{S}_{k} / \mathcal{S}_{k-1}\right)+\mu(\mathcal{L})$. Therefore

$$
\begin{aligned}
\mu_{H K}(\mathcal{S} \otimes \mathcal{L}) & =\sum_{k=1}^{t} r_{k} \mu_{k}(\mathcal{S} \otimes \mathcal{L})^{2} \\
& =\sum_{k=1}^{t} r_{k}\left(\mu_{k}+\operatorname{deg}(\mathcal{L})\right)^{2} \\
& =\sum_{k=1}^{t} r_{k}\left(\mu_{k}^{2}+2 \mu_{k} \operatorname{deg}(\mathcal{L})+\operatorname{deg}(\mathcal{L})^{2}\right)
\end{aligned}
$$




$$
=\mu_{H K}(\mathcal{S})+2 \operatorname{deg}(\mathcal{L}) \sum_{k=1}^{t} r_{k} \mu_{k}+\operatorname{deg}\left(\mathcal{L}^{2}\right) \sum_{k=1}^{t} r_{k} .
$$

This is the stated result, $\operatorname{since} \operatorname{deg}(\mathcal{S})=\sum_{k=1}^{t} r_{k} \mu_{k}$ and $\operatorname{rk}(\mathcal{S})=\sum_{k=1}^{t} r_{k}$.

(vi). The pull-back of a semistable sheaf under a separable morphism is again semistable, and the pull-back of the Harder-Narasimhan filtration is the HarderNarasimhan filtration of $\varphi^{*}(\mathcal{S})$. Hence the result follows from $\operatorname{deg}\left(\varphi^{*}(\mathcal{S})\right)=$ $n \operatorname{deg}(\mathcal{S})$.

Lemma 1.3. The Hilbert-Kunz multiplicity of a locally free sheaf $\mathcal{S}$ has the property that $\mu_{H K}(\mathcal{S}) \geq \operatorname{deg}(\mathcal{S})^{2} / \operatorname{rk}(\mathcal{S})$, and equality holds if and only if $\mathcal{S}$ is semistable.

Proof. We have to show that

$$
\sum_{k=1}^{t} r_{k} \mu_{k}^{2} \geq \operatorname{deg}(\mathcal{S})^{2} / \operatorname{rk}(\mathcal{S})=\left(r_{1} \mu_{1}+\ldots+r_{t} \mu_{t}\right)^{2} /\left(r_{1}+\ldots+r_{t}\right)
$$

or equivalently that

$$
\left(r_{1}+\ldots+r_{t}\right)\left(\sum_{k=1}^{t} r_{k} \mu_{k}^{2}\right) \geq\left(r_{1} \mu_{1}+\ldots+r_{t} \mu_{t}\right)^{2} .
$$

The left hand side is $\sum_{k=1}^{t} r_{k}^{2} \mu_{k}^{2}+\sum_{i \neq k} r_{i} r_{k} \mu_{k}^{2}$ (we sum over ordered pairs), and the right hand side is $\sum_{k=1}^{t} r_{k}^{2} \mu_{k}^{2}+\sum_{i \neq k} r_{i} r_{k} \mu_{i} \mu_{k}$. Hence left hand minus right hand is

$$
\sum_{i \neq k} r_{i} r_{k} \mu_{k}^{2}-\sum_{i \neq k} r_{i} r_{k} \mu_{i} \mu_{k}
$$

So this follows from $0 \leq\left(\mu_{i}-\mu_{k}\right)^{2}=\mu_{i}^{2}+\mu_{k}^{2}-2 \mu_{i} \mu_{k}$ for all pairs $i \neq k$. Equality holds if and only if $\mu_{i}=\mu_{k}$, but then $t=1$ and $\mathcal{S}$ is semistable.

Remark 1.4. Lemma 1.3 implies that the number $\mu_{H K}(\mathcal{S})-\frac{\operatorname{deg}(\mathcal{S})^{2}}{\operatorname{rk}(\mathcal{S})} \geq 0$, and $=0$ holds exactly in the semistable case. It follows from Proposition $1.2(\mathrm{v})$ that this number is invariant under tensoring with an invertible sheaf.

Proposition 1.5. Let $\mathcal{S}$ and $\mathcal{T}$ denote two locally free sheaves on $Y$. Then

$$
\mu_{H K}(\mathcal{S} \otimes \mathcal{T})=\operatorname{rk}(\mathcal{T}) \mu_{H K}(\mathcal{S})+\operatorname{rk}(\mathcal{S}) \mu_{H K}(\mathcal{T})+2 \operatorname{deg}(\mathcal{S}) \operatorname{deg}(\mathcal{T})
$$

Proof. Let $r_{i}, \mu_{i}, i \in I$, and $r_{j}, \mu_{j}, j \in J,(I$ and $J$ disjoint $)$ denote the ranks and slopes occurring in the Harder-Narasimhan filtration of $\mathcal{S}$ and $\mathcal{T}$ respectively. It is a non-trivial fact (in characteristic zero!) that the tensor product of two semistable bundle is again semistable, see [7, Theorem 3.1.4]. From this it follows that the semistable quotients of the Harder-Narasimhan filtration of $\mathcal{S} \otimes \mathcal{T}$ are given as $\left(\mathcal{S}_{i} / \mathcal{S}_{i-1}\right) \otimes\left(\mathcal{T}_{j} / \mathcal{T}_{j-1}\right)$ of rank $r_{i} \cdot r_{j}$ and slope $\mu_{i}+\mu_{j}$. Therefore the Hilbert-Kunz slope is

$\mu_{H K}(\mathcal{S} \otimes \mathcal{T})=\sum_{i, j} r_{i} r_{j}\left(\mu_{i}+\mu_{j}\right)^{2}$ 


$$
\begin{aligned}
& =\sum_{i, j} r_{i} r_{j} \mu_{i}^{2}+\sum_{i, j} r_{i} r_{j} \mu_{j}^{2}+2 \sum_{i, j} r_{i} r_{j} \mu_{i} \mu_{j} \\
& =\left(\sum_{j} r_{j}\right)\left(\sum_{i} r_{i} \mu_{i}^{2}\right)+\left(\sum_{i} r_{i}\right)\left(\sum_{j} r_{j} \mu_{j}^{2}\right)+2\left(\sum_{i} r_{i} \mu_{i}\right)\left(\sum_{j} r_{j} \mu_{j}\right) \\
& =\operatorname{rk}(\mathcal{T}) \mu_{H K}(\mathcal{S})+\operatorname{rk}(\mathcal{S}) \mu_{H K}(\mathcal{T})+2 \operatorname{deg}(\mathcal{S}) \operatorname{deg}(\mathcal{T})
\end{aligned}
$$

\section{A Hilbert-Kunz criterion for affine torsors}

In this section we consider a locally free sheaf $\mathcal{S}$ on a smooth projective curve $Y$ together with a cohomology class $c \in H^{1}(Y, \mathcal{S}) \cong \operatorname{Ext}\left(\mathcal{O}_{Y}, \mathcal{S}\right)$. Such a class gives rise to an extension $0 \rightarrow \mathcal{S} \rightarrow \mathcal{S}^{\prime} \rightarrow \mathcal{O}_{Y} \rightarrow 0$. Of course $\operatorname{deg}\left(\mathcal{S}^{\prime}\right)=\operatorname{deg}(\mathcal{S})$ and $\operatorname{rk}\left(\mathcal{S}^{\prime}\right)=\operatorname{rk}(\mathcal{S})+1$. We shall investigate the relationship between $\mu_{H K}(\mathcal{S})$ and $\mu_{H K}\left(\mathcal{S}^{\prime}\right)$.

Lemma 2.1. Let $Y$ denote a smooth projective curve over an algebraically closed field. Let $\mathcal{S}, \mathcal{T}$ and $\mathcal{Q}$ denote locally free sheaves on $Y$. Then the following hold.

(i) Let $\varphi: \mathcal{T} \rightarrow \mathcal{S}$ denote a sheaf homomorphism, $c \in H^{1}(Y, \mathcal{T})$ with corresponding extension $\mathcal{T}^{\prime}$, let $\mathcal{S}^{\prime}$ denote the extension of $\mathcal{S}$ corresponding to $\varphi(c) \in H^{1}(Y, \mathcal{S})$. Then there is a sheaf homomorphism $\varphi^{\prime}: \mathcal{T}^{\prime} \rightarrow \mathcal{S}^{\prime}$ extending $\varphi$.

(ii) Suppose that $0 \rightarrow \mathcal{T} \rightarrow \mathcal{S} \rightarrow \mathcal{Q} \rightarrow 0$ is a short exact sequence, and $c \in H^{1}(Y, \mathcal{T})$. Then $\mathcal{T}^{\prime} \subseteq \mathcal{S}^{\prime}$ and $\mathcal{S}^{\prime} / \mathcal{T}^{\prime} \cong \mathcal{S} / \mathcal{T}$

(iii) Suppose that $0 \rightarrow \mathcal{T} \rightarrow \mathcal{S} \rightarrow \mathcal{Q} \rightarrow 0$ is a short exact sequence, and $c \in H^{1}(Y, \mathcal{S})$. Then $\mathcal{S}^{\prime} \rightarrow \mathcal{Q}^{\prime} \rightarrow 0$ and $\mathcal{Q}^{\prime} \cong \mathcal{S}^{\prime} / \mathcal{T}$.

(iv) If $\mathcal{S}$ is semistable of degree 0 and $c \in H^{1}(Y, \mathcal{S})$, then also $\mathcal{S}^{\prime}$ is semistable.

Proof. The cohomology class $c$ is represented by the $\check{C}$ ech cocycle $\check{c} \in H^{0}\left(U_{1} \cap\right.$ $\left.U_{2}, \mathcal{S}\right)$, where $Y=U_{1} \cup U_{2}$ is an affine covering. Then $\mathcal{S}^{\prime}$ arises from $\mathcal{S}_{1}^{\prime}=$ $\left.\mathcal{S}\right|_{U_{1}} \oplus \mathcal{O}$ and $\mathcal{S}_{2}^{\prime}=\left.\mathcal{S}\right|_{U_{2}} \oplus \mathcal{O}$ by glueing $\mathcal{S}_{1}^{\prime}\left|U_{1} \cap U_{2} \cong \mathcal{S}_{2}^{\prime}\right| U_{1} \cap U_{2}$ via $(s, t) \mapsto$ $(s+t \check{c}, t)$. The natural mappings $\mathcal{T}_{i}^{\prime} \rightarrow \mathcal{S}_{i}^{\prime}, i=1,2$, glue together to a morphism $\mathcal{T}^{\prime} \rightarrow \mathcal{S}^{\prime}$. The injectivity and surjectivity transfer from $\varphi$ to $\varphi^{\prime}$, since these are local properties. (ii) and (iii) then follow from suitable diagrams.

(iv). Suppose that $\mathcal{F} \subseteq \mathcal{S}^{\prime}$ is a semistable subsheaf of positive slope. Then the induced mapping $\mathcal{F} \rightarrow \mathcal{O}$ is trivial and therefore $\mathcal{F} \subseteq \mathcal{S}$, which contradicts the semistability of $\mathcal{S}$.

Let $\mathcal{S}_{1} \subset \ldots \subset \mathcal{S}_{t}=\mathcal{S}$ denote the Harder-Narasimhan filtration of $\mathcal{S}$ and $c \in H^{1}(Y, \mathcal{S})$. If the image of $c$ in $H^{1}\left(Y, \mathcal{S} / \mathcal{S}_{t-1}\right)$ is zero, then $c$ stems from a class $c_{t-1} \in H^{1}\left(Y, \mathcal{S}_{t-1}\right)$. So we find inductively a class $c_{n} \in H^{1}\left(Y, \mathcal{S}_{n}\right)$ mapping to $c$ and such that the image in $H^{1}\left(Y, \mathcal{S}_{n} / \mathcal{S}_{n-1}\right)$ is not zero (or $c$ itself is 0 ). This yields extensions $\mathcal{S}_{k}^{\prime}$ of $\mathcal{S}_{k}$ for $k \geq n$. It is crucial for the behavior of $\mathcal{S}^{\prime}$ whether $\mu\left(\mathcal{S}_{n} / \mathcal{S}_{n-1}\right) \geq 0$ or $<0$. The following Proposition deals with the case $\mu\left(\mathcal{S}_{n} / \mathcal{S}_{n-1}\right) \geq 0$. 
Proposition 2.2. Let $\mathcal{S}_{1} \subset \ldots \subset \mathcal{S}_{t}=\mathcal{S}$ be the Harder-Narasimhan filtration of $\mathcal{S}$ and let $c \in H^{1}(Y, \mathcal{S})$. Let $n$ be such that the image of $c$ in $H^{1}\left(Y, \mathcal{S}_{k} / \mathcal{S}_{k-1}\right)$ is 0 for $k>n$ but such that the image in $H^{1}\left(Y, \mathcal{S}_{n} / \mathcal{S}_{n-1}\right)$ is $\neq 0$. Suppose that $\mu\left(\mathcal{S}_{n} / \mathcal{S}_{n-1}\right)$ is $\geq 0$. Let $i$ be the biggest number such that $\mu\left(\mathcal{S}_{i} / \mathcal{S}_{i-1}\right) \geq 0$ (hence $n \leq i)$.

(i) Suppose that $\mu_{i}>0$. Then the Harder Narasimhan filtration of $\mathcal{S}^{\prime}$ is

$$
\mathcal{S}_{1} \subset \ldots \subset \mathcal{S}_{i} \subset \mathcal{S}_{i}^{\prime} \subset \mathcal{S}_{i+1}^{\prime} \subset \ldots \subset \mathcal{S}^{\prime}
$$

(ii) Suppose that $\mu_{i}=0$. Then the Harder-Narasimhan filtration of $\mathcal{S}^{\prime}$ is

$$
\mathcal{S}_{1} \subset \ldots \subset \mathcal{S}_{i-1} \subset \mathcal{S}_{i}^{\prime} \subset \mathcal{S}_{i+1}^{\prime} \subset \ldots \subset \mathcal{S}^{\prime}
$$

Proof. (i). The quotients of the filtration are $\mathcal{S}_{k} / \mathcal{S}_{k-1}, k \leq i$, which have positive slope, $\mathcal{S}_{i}^{\prime} / \mathcal{S}_{i} \cong \mathcal{O}_{Y}$, and $\mathcal{S}_{k}^{\prime} / \mathcal{S}_{k-1}^{\prime} \cong \mathcal{S}_{k} / \mathcal{S}_{k-1}$ (Lemma 2.1 (ii)) for $k>i$, which have negative slope. These quotients are all semistable and the slope numbers are decreasing.

(ii). The quotients $\mathcal{S}_{k} / \mathcal{S}_{k-1}$ are semistable with decreasing positive slopes for $k=1, \ldots, i-1$. The quotients $\mathcal{S}_{k}^{\prime} / \mathcal{S}_{k-1}^{\prime} \cong \mathcal{S}_{k} / \mathcal{S}_{k-1}$ are semistable with decreasing negative slopes for $k=i+1, \ldots, t$. The quotient $\mathcal{S}_{i}^{\prime} / \mathcal{S}_{i-1}$ is isomorphic to $\left(\mathcal{S}_{i} / \mathcal{S}_{i-1}\right)^{\prime}$ by Lemma 2.1 (iii), hence semistable of degree 0 by Lemma 2.1 (iv).

In the rest of this section we study the remaining case, that $\mu\left(\mathcal{S}_{n} / \mathcal{S}_{n-1}\right)<0$. In this case it is not possible to describe the Harder-Narasimhan filtration of $S^{\prime}$ explicitly. However we shall see that in this case the Hilbert-Kunz slope of $\mathcal{S}^{\prime}$ is smaller than the Hilbert-Kunz slope of $\mathcal{S}$. We need the following two lemmata.

Lemma 2.3. Let $\mathcal{T}$ denote a locally free sheaf on $Y$ with Harder-Narasimhan filtration $\mathcal{T}_{k}, \mu_{k}=\mu\left(\mathcal{T}_{k} / \mathcal{T}_{k-1}\right)$ and $r_{k}=\operatorname{rk}\left(\mathcal{T}_{k} / \mathcal{T}_{k-1}\right)$. Let

$$
\left(\tau_{i}\right)=\left(\mu_{1}, \ldots, \mu_{1}, \mu_{2}, \ldots, \mu_{2}, \mu_{3}, \ldots, \mu_{t-1}, \mu_{t}, \ldots, \mu_{t}\right)
$$

denote the slopes where each $\mu_{k}$ occurs $r_{k}$-times. Let $\mathcal{S} \subseteq \mathcal{T}$ denote a locally free subsheaf of rank $r$ and let $\sigma_{i}, i=1, \ldots, r$ denote the corresponding numbers for $\mathcal{S}$. Then $\sigma_{i} \leq \tau_{i}$ for $i=1, \ldots, r$.

Moreover, if $\mathcal{S}$ is saturated (meaning that the quotient sheaf is locally free) and if no subsheaf $\mathcal{S}_{j}$ of the Harder-Narasimhan filtration of $\mathcal{S}$ occurs in the Harder-Narasimhan filtration of $\mathcal{T}$, then $\sigma_{i} \leq \tau_{i+1}$ for $i=1, \ldots, r$.

Proof. Let $i, i=1, \ldots, r$ be given and let $j$ be such that $\operatorname{rk}\left(\mathcal{S}_{j-1}\right)<i \leq \operatorname{rk}\left(\mathcal{S}_{j}\right)$, hence $\sigma_{i}=\mu_{j}(\mathcal{S})=\mu\left(\mathcal{S}_{j} / \mathcal{S}_{j-1}\right)$. We may assume that $i=\operatorname{rk}\left(\mathcal{S}_{j}\right)$. Let $k$ be such that $\operatorname{rk}\left(\mathcal{T}_{k-1}\right)<i \leq \operatorname{rk}\left(\mathcal{T}_{k}\right)$. Therefore $\mathcal{S}_{j} \nsubseteq \mathcal{T}_{k-1}$, and the induced morphism $\mathcal{S}_{j} \rightarrow \mathcal{T} / \mathcal{T}_{k-1}$ is not trivial. Hence $\sigma_{i}=\mu_{j}(\mathcal{S})=\mu_{\min }\left(\mathcal{S}_{j}\right) \leq \mu_{\max }\left(\mathcal{T} / \mathcal{T}_{k-1}\right)=$ $\mu_{k}(\mathcal{T})=\tau_{i}$.

Now suppose that $\sigma_{i}>\tau_{i+1}$. Then necessarily $\sigma_{i}>\sigma_{i+1}$ and $\tau_{i}>\tau_{i+1}$ by what we have already proven. Therefore $i=\operatorname{rk}\left(\mathcal{S}_{j}\right)=\operatorname{rk}\left(\mathcal{T}_{k}\right)$. If $\mathcal{S}_{j} \subseteq \mathcal{T}_{k}$, then they are equal, since both sheaves are saturated of the same rank, but this is excluded by the assumptions. Hence $\mathcal{S}_{j} \nsubseteq \mathcal{T}_{k}$ and $\mathcal{S}_{j} \rightarrow \mathcal{T} / \mathcal{T}_{k}$ is non-trivial. Therefore $\sigma_{i}=\mu_{\min }\left(\mathcal{S}_{j}\right) \leq \mu_{\max }\left(\mathcal{T} / \mathcal{T}_{k}\right)=\mu_{k+1}(\mathcal{T})=\tau_{i+1}$. 
Remark 2.4. If the numbers $\tau_{i}$ are given as in the previous lemma, then $\operatorname{deg}(\mathcal{T})$ $=\sum_{i} \tau_{i}$ and $\mu_{H K}(\mathcal{T})=\sum_{i} \tau_{i}^{2}$.

Lemma 2.5. Let $\alpha_{1} \leq \ldots \leq \alpha_{r}$ and $\beta_{1} \leq \ldots \leq \beta_{r+1}$ denote non-negative real numbers such that $\alpha_{i} \geq \beta_{i+1}$ for $i=1, \ldots, r$ and $\sum_{i=1}^{r} \alpha_{i}=\sum_{i=1}^{r+1} \beta_{i}$. Then $\sum_{i=1}^{r+1} \beta_{i}^{2} \leq \sum_{i=1}^{r} \alpha_{i}^{2}$ and equality holds if and only if $\alpha_{i}=\beta_{i+1}$.

Proof. Let $\alpha_{i}=\beta_{i+1}+\delta_{i}, \delta_{i} \geq 0$. From $\sum_{i=1}^{r} \alpha_{i}=\sum_{i=1}^{r} \delta_{i}+\sum_{i=1}^{r} \beta_{i+1}=$ $\sum_{i=1}^{r+1} \beta_{i}$ we get $\beta_{1}=\sum_{i=1}^{r} \delta_{i}\left(\leq \beta_{2}\right)$. The quadratic sums are

$$
\sum_{i=1}^{r} \alpha_{i}^{2}=\sum_{i=2}^{r+1} \beta_{i}^{2}+\sum_{i=1}^{r} \delta_{i}^{2}+2 \sum_{i=1}^{r} \delta_{i} \beta_{i+1}
$$

and

$$
\sum_{i=1}^{r+1} \beta_{i}^{2}=\left(\sum_{i=1}^{r} \delta_{i}\right)^{2}+\sum_{i=2}^{r+1} \beta_{i}^{2}=2 \sum_{i<j} \delta_{i} \delta_{j}+\sum_{i=1}^{r} \delta_{i}^{2}+\sum_{i=2}^{r+1} \beta_{i}^{2} .
$$

So we have to show that $\sum_{i<j} \delta_{i} \delta_{j} \leq \sum_{j=1}^{r} \delta_{i} \beta_{i+1}$. But this is clear from $\sum_{i<j} \delta_{j} \leq \sum_{j=1}^{r} \delta_{j} \leq \beta_{2} \leq \beta_{i+1}$ for all $i=1, \ldots, r$. Equality holds if and only if $\delta_{i}=0$.

A cohomology class $H^{1}(Y, \mathcal{S})$ corresponds to a geometric $\mathcal{S}$-torsor $T \rightarrow Y$. This is an affine-linear bundle on which $\mathcal{S}$ acts transitively. A geometric realization is given as $T=\mathbb{P}\left(\mathcal{S}^{\prime \vee}\right)-\mathbb{P}\left(\mathcal{S}^{\vee}\right)$. The global cohomological properties of this torsor are related to the Hilbert-Kunz slope in the following way.

Theorem 2.6. Let $Y$ denote a smooth projective curve over an algebraically closed field of characteristic 0 . Let $\mathcal{S}$ denote a locally free sheaf on $Y$ and let $c \in H^{1}(Y, \mathcal{S})$ denote a cohomology class given rise to the extension $0 \rightarrow \mathcal{S} \rightarrow$ $\mathcal{S}^{\prime} \rightarrow \mathcal{O}_{Y} \rightarrow 0$ and the affine-linear torsor $\mathbb{P}\left(\mathcal{S}^{\prime \vee}\right)-\mathbb{P}\left(\mathcal{S}^{\vee}\right)$. Then the following are equivalent.

(i) There exists a locally free quotient $\varphi: \mathcal{S} \rightarrow \mathcal{Q} \rightarrow 0$ such that $\mu_{\max }(\mathcal{Q})<0$ and the image $\varphi(c) \in H^{1}(Y, \mathcal{Q})$ is non-trivial.

(ii) The torsor $\mathbb{P}\left(\mathcal{S}^{\prime \vee}\right)-\mathbb{P}\left(\mathcal{S}^{\vee}\right)$ is an affine scheme.

(iii) The Hilbert-Kunz slope drops, that is $\mu_{H K}\left(\mathcal{S}^{\prime}\right)<\mu_{H K}(\mathcal{S})$.

Proof. The equivalence (i) $\Leftrightarrow$ (ii) was shown in [3, Theorem 2.3]. The implication (iii) $\Rightarrow$ (i) follows from Proposition 2.2: for if (i) does not hold, then we are in the situation of Proposition 2.2 that $\mu\left(\mathcal{S}_{n} / \mathcal{S}_{n-1}\right) \geq 0$. The explicit description of the Harder-Narasimhan filtration of $\mathcal{S}^{\prime}$ gives in both cases that $\mu_{H K}\left(\mathcal{S}^{\prime}\right)=\mu_{H K}(\mathcal{S})$.

So suppose that (i) holds. This means that there exists a subsheaf $\mathcal{S}_{n} \subseteq$ $\mathcal{S}$ occurring in the Harder-Narasimhan filtration of $\mathcal{S}$ such that $c$ stems from $c_{n} \in H^{1}\left(Y, \mathcal{S}_{n}\right)$ and such that its image in $H^{1}\left(Y, \mathcal{S} / \mathcal{S}_{n-1}\right)$ is non-trivial with $\mu_{\max }\left(\mathcal{S} / \mathcal{S}_{n-1}\right)=\mu\left(\mathcal{S}_{n} / \mathcal{S}_{n-1}\right)=\mu_{n}<0$.

Let $\mathcal{T}_{1} \subset \ldots \subset \mathcal{T}_{t}=\mathcal{S}^{\prime}$ denote the Harder-Narasimhan filtration of $\mathcal{S}^{\prime}$ with slopes $\mu_{k}=\mu\left(\mathcal{T}_{k} / \mathcal{T}_{k-1}\right)$ and ranks $r_{k}=\operatorname{rk}\left(\mathcal{T}_{k} / \mathcal{T}_{k-1}\right)$. Suppose that the maximal slope $\mu\left(\mathcal{T}_{1}\right)$ is positive. Then the induced mapping $\mathcal{T}_{1} \rightarrow \mathcal{S}^{\prime} / \mathcal{S} \cong \mathcal{O}_{Y}$ is trivial, 
and $\mathcal{T}_{1} \subseteq \mathcal{S}$. This is then also the maximal destabilizing subsheaf of $\mathcal{S}$, since $\mu_{\max }(\mathcal{S}) \leq \mu_{\max }\left(\mathcal{S}^{\prime}\right)=\mu\left(\mathcal{T}_{1}\right)$. Therefore $\mu_{H K}(\mathcal{S})=\mu_{H K}\left(\mathcal{T}_{1}\right)+\mu_{H K}\left(\mathcal{S} / \mathcal{T}_{1}\right)$ and $\mu_{H K}\left(\mathcal{S}^{\prime}\right)=\mu_{H K}\left(\mathcal{T}_{1}\right)+\mu_{H K}\left(\mathcal{S}^{\prime} / \mathcal{T}_{1}\right)$ by Proposition $1.2\left(\right.$ ii). Since $\mathcal{S}^{\prime} / \mathcal{T}_{1}$ is the extension of $\mathcal{S} / \mathcal{T}_{1}$ defined by the image of the cohomology class in $H^{1}\left(Y, \mathcal{S} / \mathcal{T}_{1}\right)$ (Lemma 2.1(iii)), we may mod out $\mathcal{T}_{1}$. Note that this does not change the condition in (i). Hence we may assume inductively that $\mu_{\max }(\mathcal{S}) \leq 0$ and $\mu_{\max }\left(\mathcal{S}^{\prime}\right) \leq 0$.

Now suppose that $\mathcal{T}_{1}$ has degree 0 . Again, if $\mathcal{T}_{1} \subseteq \mathcal{S}$, then this is also the maximal destabilizing subsheaf of $\mathcal{S}$, and we can mod out $\mathcal{T}_{1}$ as before. So suppose that $\mathcal{T}_{1} \rightarrow \mathcal{O}_{Y}$ is non-trivial. Then this mapping is surjective, let $\mathcal{K} \subset \mathcal{S}$ denote the kernel. This means that the extension defined by $c \in H^{1}(Y, \mathcal{S})$ comes from the extension given by $0 \rightarrow \mathcal{K} \rightarrow \mathcal{T}_{1} \rightarrow \mathcal{O}_{Y} \rightarrow 0$, and $\tilde{c} \in H^{1}(Y, \mathcal{K}) . \mathcal{K}$ is semistable, since its degree is 0 and $\mu_{\max }(\mathcal{S}) \leq 0$. But then the image of $c$ is 0 in every quotient sheaf of $\mathcal{S}$ with negative maximal slope, which contradicts the assumptions. Therefore we may assume that $\mu_{\max }\left(\mathcal{S}^{\prime}\right)<0$.

We want to apply Lemma 2.3 to $\mathcal{S} \subset \mathcal{S}^{\prime}=\mathcal{T}$. Assume that $\mathcal{S}$ and $\mathcal{S}^{\prime}$ have a common subsheaf occuring in both Harder-Narasimhan filtrations. Then they have the same maximal destabilizing subsheaf $\mathcal{F}=\mathcal{S}_{1}=\mathcal{T}_{1}$, which has negative degree. If $c$ comes from $\tilde{c} \in H^{1}(Y, \mathcal{F})$, then $\mathcal{F} \subset \mathcal{F}^{\prime} \subseteq \mathcal{S}^{\prime}$ and $\mu(\mathcal{F})=$ $\operatorname{deg}(\mathcal{F}) / \operatorname{rk}(\mathcal{F})<\operatorname{deg}(\mathcal{F}) /(\operatorname{rk}(\mathcal{F})+1)=\mu\left(\mathcal{F}^{\prime}\right)$, which contradicts the maximality of $\mathcal{F}$. Hence the image of $c$ in $H^{1}(Y, \mathcal{S} / \mathcal{F})$ is not zero and we can mod out $\mathcal{F}$ as before.

Therefore we may assume that $\mathcal{S}$ and $\mathcal{S}^{\prime}$ do not have any common subsheaf in their Harder-Narasimhan filtrations. Then Lemma 2.3 yields that $\sigma_{i} \leq \tau_{i+1}$, and all these numbers are $\leq 0$ and moreover $\tau_{i}<0$. Lemma 2.5 applied to $\alpha_{i}=-\sigma_{i}$ and $\beta_{i}=-\tau_{i}$ yields that $\sum_{i=1}^{r} \sigma_{i}^{2} \geq \sum_{i=1}^{r+1} \tau_{i}^{2}$, and $>$ holds since $\tau_{1} \neq 0$.

Remark 2.7. Suppose that $\mathcal{S}$ is a semistable locally free sheaf of negative degree, and let $c \in H^{1}(Y, \mathcal{S})$ with corresponding extension $\mathcal{S}^{\prime}$. Then Theorem 2.6 together with Lemma 1.3 yield the inequalities

$$
\frac{\operatorname{deg}(\mathcal{S})^{2}}{r+1} \leq \mu_{H K}\left(\mathcal{S}^{\prime}\right) \leq \frac{\operatorname{deg}(\mathcal{S})^{2}}{r}
$$

If $\mathcal{S}^{\prime}$ is also semistable, then we have equality on the left.

\section{A Hilbert-Kunz criterion for solid closure}

We come now back to our original setting of interest, that of a two-dimensional normal standard-graded domain $R$ over an algebraically closed field $K$. A homogeneous $R_{+}$-primary ideal $I=\left(f_{1}, \ldots, f_{n}\right)$ gives rise to the syzygy bundle $\operatorname{Syz}\left(f_{1}, \ldots, f_{n}\right)(0)$ on $Y=\operatorname{Proj} R$ defined by the presenting sequence

$$
0 \longrightarrow \operatorname{Syz}\left(f_{1}, \ldots, f_{n}\right)(m) \longrightarrow \bigoplus_{i=1}^{n} \mathcal{O}_{Y}\left(m-d_{i}\right) \stackrel{f_{1}, \ldots, f_{n}}{\longrightarrow} \mathcal{O}_{Y}(m) \longrightarrow 0
$$


Another homogeneous element $f$ of degree $m$ yields an extension

$$
0 \longrightarrow \operatorname{Syz}\left(f_{1}, \ldots, f_{n}\right)(m) \longrightarrow \operatorname{Syz}\left(f_{1}, \ldots, f_{n}, f\right)(m) \longrightarrow \mathcal{O}_{Y} \longrightarrow 0
$$

which corresponds to the cohomology class $\delta(f) \in H^{1}\left(Y, \operatorname{Syz}\left(f_{1}, \ldots, f_{n}\right)(m)\right)$ coming from the presenting sequence via the connecting homomorphism

$$
\delta: H^{0}\left(Y, \mathcal{O}_{Y}(m)\right)=R_{m} \rightarrow H^{1}\left(Y, \operatorname{Syz}\left(f_{1}, \ldots, f_{n}\right)(m)\right) .
$$

The Hilbert-Kunz multiplicities of the ideals and the Hilbert-Kunz slopes of the syzygy bundles are related in the following way.

Lemma 3.1. Let $K$ denote an algebraically closed field of characteristic 0 . Let $R$ denote a standard-graded two-dimensional normal $K$-domain, $Y=\operatorname{Proj} R$. Let $I$ be a homogeneous $R_{+}$-primary ideal and let $f$ denote a homogeneous element of degree $m$. Then the Hilbert-Kunz multiplicities $e_{H K}(I)=e_{H K}((I, f))$ are equal if and only if the Hilbert-Kunz slopes of the corresponding syzygies bundles $\mu_{H K}\left(\operatorname{Syz}\left(f_{1}, \ldots, f_{n}\right)(m)\right)=\mu_{H K}\left(\operatorname{Syz}\left(f_{1}, \ldots, f_{n}, f\right)(m)\right)$ are equal.

Proof. Let $\mu_{k}$ and $r_{k}\left(\tilde{\mu}_{k}\right.$ and $\left.\tilde{r}_{k}\right)$ denote the ranks and the slopes in the Harder-Narasimhan filtration of $\operatorname{Syz}\left(f_{1}, \ldots, f_{n}\right)(0)$ (of $\operatorname{Syz}\left(f_{1}, \ldots, f_{n}, f\right)(0)$ respectively). For the Hilbert-Kunz multiplicities of the ideals $\left(f_{1}, \ldots, f_{n}\right)$ and $\left(f_{1}, \ldots, f_{n}, f\right)$ we have to compare

$$
e_{H K}(I)=\frac{1}{2 \operatorname{deg}(Y)}\left(\sum_{k=1}^{t} r_{k} \mu_{k}^{2}-\operatorname{deg}(Y)^{2} \sum_{i=1}^{n} d_{i}^{2}\right)
$$

and

$$
e_{H K}((I, f))=\frac{1}{2 \operatorname{deg}(Y)}\left(\sum_{k=1}^{\tilde{t}} \tilde{r}_{k} \tilde{\mu}_{k}^{2}-\operatorname{deg}(Y)^{2}\left(m^{2}+\sum_{i=1}^{n} d_{i}^{2}\right)\right) .
$$

The extension defined by $c=\delta(f) \in H^{1}\left(Y, \operatorname{Syz}\left(f_{1}, \ldots, f_{n}\right)(m)\right)$ is

$$
0 \longrightarrow \mathcal{S}=\operatorname{Syz}\left(f_{1}, \ldots, f_{n}\right)(m) \longrightarrow \mathcal{S}^{\prime}=\operatorname{Syz}\left(f_{1}, \ldots, f_{n}, f\right)(m) \longrightarrow \mathcal{O}_{Y} \longrightarrow 0
$$

and the Hilbert-Kunz slopes of these sheaves are due to Proposition $1.2(\mathrm{v})$ (since $\left.\operatorname{deg}\left(\operatorname{Syz}\left(f_{1}, \ldots, f_{n}\right)(0)\right)=-\operatorname{deg}(Y) \sum_{i=1}^{n} d_{i}\right)$

$$
\mu_{H K}(\mathcal{S})=\sum_{k=1}^{t} r_{k} \mu_{k}^{2}+2\left(-\sum_{i=1}^{n} d_{i} \operatorname{deg}(Y)\right) m \operatorname{deg}(Y)+(n-1) m^{2} \operatorname{deg}(Y)^{2}
$$

and $\mu_{H K}\left(\mathcal{S}^{\prime}\right)=$

$$
\begin{aligned}
& =\sum_{k=1}^{\tilde{t}} \tilde{r}_{k} \tilde{\mu}_{k}^{2}+2\left(-\left(\sum_{i=1}^{n} d_{i}+m\right) \operatorname{deg}(Y)\right) m \operatorname{deg}(Y)+n m^{2} \operatorname{deg}(Y)^{2} \\
& =\sum_{k=1}^{\tilde{t}} \tilde{r}_{k} \tilde{\mu}_{k}^{2}-2\left(\sum_{i=1}^{n} d_{i}\right) m \operatorname{deg}(Y)^{2}+(n-1) m^{2} \operatorname{deg}(Y)^{2}-m^{2} \operatorname{deg}(Y)^{2} .
\end{aligned}
$$


So the difference is in both cases (up to the factor $1 / 2 \operatorname{deg}(Y)$ )

$$
\sum_{k=1}^{\tilde{t}} \tilde{r}_{k} \tilde{\mu}_{k}^{2}-\sum_{k=1}^{t} r_{k} \mu_{k}^{2}-m^{2} \operatorname{deg}(Y)^{2} .
$$

Therefore $e_{H K}(I)=e_{H K}((I, f))$ if and only if

$$
\mu_{H K}\left(\operatorname{Syz}\left(f_{1}, \ldots, f_{n}\right)(m)\right)=\mu_{H K}\left(\operatorname{Syz}\left(f_{1}, \ldots, f_{n}, f\right)(m)\right) .
$$

Remark 3.2. Let $0 \rightarrow \mathcal{S} \rightarrow \mathcal{T} \rightarrow \mathcal{Q} \rightarrow 0$ denote a short exact sequence of locally free sheaves. Then the alternating sum of the Hilbert-Kunz slopes, that ist $\mu_{H K}(\mathcal{S})-\mu_{H K}(\mathcal{T})+\mu_{H K}(\mathcal{Q})$ does not change when we tensor the sequence with an invertible sheaf. This follows from Proposition 1.2(v). For an extension $0 \rightarrow \mathcal{S} \rightarrow \mathcal{S}^{\prime} \rightarrow \mathcal{O}_{Y} \rightarrow 0$ this number is $\geq 0$ by Theorem 2.6, and we suspect that this is true in general. From the presenting sequence $0 \rightarrow \operatorname{Syz}\left(f_{1}, \ldots, f_{n}\right)(0) \rightarrow \bigoplus_{i=1}^{n} \mathcal{O}\left(-d_{i}\right) \rightarrow \mathcal{O}_{Y} \rightarrow 0$ it follows via $e_{H K}(I)=$ $\frac{1}{2 \operatorname{deg}(Y)}\left(\mu_{H K}\left(\operatorname{Syz}\left(f_{1}, \ldots, f_{n}\right)(0)\right)-\mu_{H K}\left(\bigoplus_{i=1}^{n} \mathcal{O}\left(-d_{i}\right)\right)\right.$ that the Hilbert-Kunz multiplicity of an ideal is always nonnegative. In fact $I=R$ is the only ideal with $e_{H K}(I)=0$. This follows from Theorem 3.3 below, since $1 \notin I^{\star}$ for $I \neq R$.

We come now to the main result of this paper. Recall that the solid closure of an $\mathfrak{m}$-primary ideal $I=\left(f_{1}, \ldots, f_{n}\right)$ in a two-dimensional normal excellent domain $R$ is given by the condition that $f \in\left(f_{1}, \ldots, f_{n}\right)^{\star}$ if and only $D(\mathfrak{m}) \subset$ Spec $R\left[T_{1}, \ldots, T_{n}\right] /\left(f_{1} T_{1}+\ldots+f_{n} T_{n}+f\right)$ is not an affine scheme. In positive characteristic this is the same as tight closure, see [5, Theorem 8.6]. In the case of an $R_{+}$-primary homogeneous ideal in a standard-graded normal $K$-domain this is equivalent to the property that the torsor $\mathbb{P}\left(\mathcal{S}^{\vee}\right)-\mathbb{P}\left(\mathcal{S}^{\vee}\right)$ over the corresponding curve $Y=\operatorname{Proj} R$ is not affine (see [1, Proposition 3.9]). This relates solid closure to the setting of the previous section.

Theorem 3.3. Let $K$ denote an algebraically closed field. Let $R$ denote a standard-graded two-dimensional normal $K$-domain. Let $I$ be a homogeneous $R_{+}$-primary ideal and let $f$ denote a homogeneous element. Then $f \in I^{\star}$ if and only if $e_{H K}(I)=e_{H K}((I, f))$.

Proof. If the characteristic is positive then this is a standard result from tight closure theory as mentioned in the introduction. So suppose that the characteristic is 0 . Let $I=\left(f_{1}, \ldots, f_{n}\right)$ be generated by homogeneous elements, and set $m=\operatorname{deg}(f)$. The containment in the solid closure, $f \in\left(f_{1}, \ldots, f_{n}\right)^{\star}$, is equivalent with the non-affineness of the torsor $\mathbb{P}\left(\mathcal{S}^{\prime \vee}\right)-\mathbb{P}\left(\mathcal{S}^{\vee}\right)[1$, Proposition 3.9], where $\mathcal{S}=\operatorname{Syz}\left(f_{1}, \ldots, f_{n}\right)(m)$ and $S^{\prime}$ is the extension given by the cohomology class $\delta(f)$. Hence the result follows from Theorem 2.6 and Lemma 3.1.

\section{References}

[1] H. Brenner, Tight closure and projective bundles, J. Algebra, 256 (2003), 45-78. 
[2] — The rationality of the Hilbert-Kunz multiplicity in graded dimension two, ArXiv, (2004).

[3] _ Tight closure and plus closure in dimension two, ArXiv, (2004).

[4] G. Harder and M. S. Narasimhan, On the cohomology groups of moduli spaces of vector bundles on curves, Math. Ann., 212 (1975), 215-248.

[5] M. Hochster, Solid closure, Contemp. Math., 159 (1994), 103-172.

[6] C. Huneke, Tight closure and its applications, AMS, (1996).

[7] D. Huybrechts and M. Lehn, The geometry of moduli spaces of sheaves, Veihweg, (1997).

[8] A. Langer, Semistable sheaves in positive characteristic, Ann. Math., 159 (2004), 251276.

[9] P. Monsky, The Hilbert-Kunz function, Math. Ann., 263 (1983), 43-49.

[10] V. Trivedi, Semistability and Hilbert-Kunz multiplicity for curves, ArXiv, (2004).

Department of Pure Mathematics, University of Sheffield, The Hicks Building, Hounsfield Road, Sheffield, UK

E-mail address: H.Brenner@sheffield.ac.uk 